\title{
Ribosomal protein S15A promotes malignant transformation and predicts poor outcome in colorectal cancer through misregulation of $\mathbf{p 5 3}$ signaling pathway
}

\author{
JINGWEN CHEN*, YE WEI*, QINGYANG FENG* , LI REN, GUODONG HE, WENJU CHANG, \\ DEXIANG ZHU, TUO YI, QI LIN, WENTAO TANG, JIANMIN XU and XINYU QIN \\ Department of General Surgery, Zhongshan Hospital, Fudan University, Shanghai 200032, P.R. China
}

Received December 3, 2015; Accepted January 5, 2016

DOI: $10.3892 /$ ijo.2016.3366

\begin{abstract}
Ribosomal protein S15A (RPS15A), which has been identified as a highly conserved $40 \mathrm{~S}$ ribosomal protein, is essential for cell survival and proliferation. The present study evaluated the functional role of RPS15A in colorectal cancer (CRC), and our investigation found that RPS15A was highly expressed in a cohort of human CRC. High RPS15A expression was associated with older age $(\mathrm{P}=0.035)$, not receiving preoperative neoadjuvant treatment $(\mathrm{P}=0.048)$, higher primary pN stage $(\mathrm{P}=0.007)$ and slightly more synchronous distant metastases $(\mathrm{P}=0.058)$. The Cox univariate and multivariate hazard regression analysis revealed that higher expression of RPS15A led to a reduction of overall survival rate in CRC, indicating that enhanced RPS15A expression functions as an independent risk factor for the prognosis of CRC patients $(\mathrm{P}<0.001)$. Cell based analysis showed that RPS15A was widely expressed in human CRC cell lines. Knockdown of RPS15A significantly suppressed cell proliferation and colony formation in HCT116 and DLD-1 cells, and induced cell cycle arrest at G0/G1 phase. GeneChip analysis suggested that knockdown of RPS15A might affect the p53 signaling pathway. Further study indicated that RPS15A knockdown upregulated p53 and p21 expression whereas downregulated CDK1 expression. In summary, the present study identified RPS15A as a novel univariate prognostic factor predicting a poor outcome in CRC patients. The RPS15A overexpression induced by malignant transformation of CRC might function through the p53 signaling pathway.
\end{abstract}

Correspondence to: Dr Jianmin Xu or Dr Xinyu Qin, Department of General Surgery, Zhongshan Hospital, Fudan University, No. 180 Fenglin Road, Shanghai 200032, P.R. China

E-mail:xujmin@aliyun.com

E-mail: qin.xinyu@zs-hospital.sh.cn

*Contributed equally

Key words: colorectal cancer, ribosomal protein S15A, cell cycle, p53

\section{Introduction}

Colorectal cancer (CRC) is one of the leading causes of death in western countries (1), and the incidence is still increasing. Approximately $70 \%$ of patients with CRC are $>65$ years while the disease is rare under the age of 45 (2). Advancements in surgery and perioperative therapies have significantly improved the survival rate in patients with CRC (3). However, the possibility of tumor recurrence remains high even after surgical therapy and it depends on the stage of cancer at the time of diagnosis. Recently, molecular targeting, a novel therapeutic strategy, has been widely researched and applied in the treatment of CRC.

Ribosomal proteins (RPs) play an important role in forming the majority of cellular proteins and are considered to be indispensable for cell growth (4). RP genes have been linked, either directly or indirectly, to various diseases including cancer. Eukaryotic ribosomes are composed of $\sim 79$ different ribosomal proteins (5). Even though the functions of RPs have not been fully investigated, quantitative deficiencies of RP genes have been suggested to contribute to the growth retardation and abnormalities (6,7). Ribosomal protein S15A (RPS15A) is a highly conserved 40S ribosomal protein which promotes the binding of capped mRNA to the small ribosomal subunit at the early stages of translation (8). RPS15A is found to be a responsive gene of transforming growth factor beta (TGF- $\beta$ ) (9). In addition, RPS15A has been reported to promote cell division in actively dividing tissues. In humans, the expression of RPS15A is closely related with cancer progression. Accumulated evidence shows that RPS15A could stimulate cell growth and proliferation in hepatocellular carcinoma (10) and lung adenocarcinoma (11). However, its prognostic significance in CRC remains unclear and there is scarce previous information concerning the possible role of RPS15A in human CRC.

In our pilot project, in a lentivirus-based screening of CRC related genes, we identified RPS15A as a potential oncogene in $\mathrm{CRC}$. To further demonstrate the hypothesis, we first studied the correlation between RPS15A expression and the clinicopathological features and prognosis in CRC patients, and then investigated the effects and possible mechanisms of RNAi mediated RPS15A gene knockdown on CRC cells. 


\section{Materials and methods}

Patient tissues and cell lines. Tumor tissues and their corresponding normal tissues from 200 consecutive patients were prepared for immunohistochemistry (IHC) assay. According to the criteria of the World Health Organization classification, the tumor-node-metastasis (TNM) stage was determined. All specimens were collected at the Department of General Surgery, Zhongshan Hospital, Fudan University in Shanghai between January 2008 and December 2009. All patients were then followed-up. All participants provided written informed consent. The present study was approved by the ethics committee of the Zhongshan Hospital, Fudan University.

Human CRC cell lines DLD-1, HCT116, HT-29, RKO, SW1116, SW480 and human embryonic kidney cell line HEK293T were obtained from the Cell Bank of Chinese Academy of Sciences (Shanghai, China). DLD-1, RKO, SW1116 and SW480 cells were maintained in RPMI-1640 medium (Hyclone Laboratories, Logan, UT, USA) supplemented with $10 \%$ heat inactivated fetal bovine serum (FBS). HCT116 and HT-29 cells were maintained in McCoy's 5A (Sigma) supplemented with $10 \%$ heat inactivated FBS. HEK293T cells were cultured in Dulbecco's modified Eagle's medium (DMEM; HyClone Laboratories) supplemented with $10 \%$ heat inactivated FBS. All cells were incubated at $37^{\circ} \mathrm{C}$ in humidified atmosphere of $5 \% \mathrm{CO}_{2}$.

Immunohistochemistry (IHC). For formalin-fixed paraffinembedded (FFPE) tissue sections, Histostain-Plus 3rd Gen IHC detection kit (85-9073; Invitrogen, Carlsbad, CA, USA) was used to perform IHC assay according to the manufacturer's instructions. IHC score was used to evaluate RPS15A expression in these tissues. IHC score was calculated as the multiplication product of two independent scores: the proportion of positive tumor cells in the tissues as $0(0 \%), 1(0-20 \%)$, $2(20-60 \%), 3(60-100 \%)$, respectively; and the score of the intensity of positive tumor cells in the tumor tissues as 0 (negative), 1 (yellow), 2 (faint yellow), 3 (reddish brown), respectively. For IHC score, the cut-off for the definition of high/low expression subgroups was the median value.

Lentivirus vectors construction and infection. To silence RPS15A expression, two short hairpin (shRNA) sequences were identified to target human RPS15A gene (NM_001019). The shRNA sequences are S1, 5'-GTGCAACTCAAAGA CCTGGAACTCGAGTTCCAGGTCTTTGAGTTGCACTT TTT-3' and S2, 5'-GCATGGTTACATTGGCGAATTCTC GAGAATTCGCCAATGTAACCATGCTTTTT-3'. The nonsilencing shRNA (5'-GCGGAGGGTTTGAAAGAATATCT CGAGATATTCTTTCAA ACCCTCCGCTTTTTT-3') was used as control. The stem-loop-stem oligos were synthesized, annealed, and ligated into the NheI/PacI-linearized pFH-L vector (Shanghai Hollybio, Shanghai, China). The reconstructed pFH-L-shRPS15A and pFH-L-shCon vectors were co-transfected into HEK293T cells with packing helper plasmid spVSVG-I and pCMVAR8.92 (Shanghai Hollybio) to generate lentiviruses. After $96-\mathrm{h}$ incubation, the lentiviral particles were harvested from the supernatant by ultracentrifugation. The RNAi lentiviruses were referred as Lv-shRPS15A (KD) for specific RPS15A gene knockdown and Lv-shCon
(NC) for control. For lentiviral transduction, HCT116 cells $\left(8 \times 10^{4}\right.$ cells/well) and DLD-1 cells $\left(5 \times 10^{4}\right.$ cells/well) were seeded onto 6-well plates and incubated with $\mathrm{NC}$ or KD for $96 \mathrm{~h}$, respectively, with a replacement of media $24 \mathrm{~h}$ after lentivirus transduction.

RNA extraction and real-time PCR analysis. After 96-h incubation, total RNA was isolated from cultured cells by the TRIzol ${ }^{\circledR}$ reagent, and cDNA was synthesized from the extracted RNA using Promega M-MLV cDNA synthesis kit according to the manufacturer's instructions. RPS15A mRNA level was evaluated by real-time PCR using the SYBR Premix Ex Taq ${ }^{\mathrm{TM}}$ Perfect Real-Time (Takara Bio, Shiga, Japan) on an ABI PRISM 7500 Real-Time system. $\beta$-actin gene was applied as the input reference. The primers used for real-time PCR are listed as follows: RPS15A-forward, 5'-TGACGTGCAACTCAAAGACC-3' and RPS15A-reverse, 5'-CCAGAGTCCATGAGGCATTT-3'; DUSP6-forward, 5'-CAGCGACTGGAACGAGAATAC-3' and DUSP6-reverse, 5'-ACTCGATGTCCGAGGAAGAGT-3'; p38-forward, 5'-GCTTGCGACTCACAGGATTG-3' and p38-reverse, 5'-GAGAAATTGCCCTCTGAACCC-3'; p53-forward, 5'-CACACCCTGGAGGATTTCATC-3' and p53-reverse, 5'-GGGCAACAAAGCGAGACC-3'; 14-3-3 $\alpha$-forward, 5'-AAGAGCGAAACCTGCTCTCA-3' and 14-3-3 $\alpha$-reverse, 5'-CTCCACCTTCTCCCGGTACT-3'; CDK1-forward, 5'-TG GAGTTGTGTATAAGGGTAGAC-3' and CDK1-reverse, 5'-GATGACGAAGTTCCTTTAATAGAG-3'; p21-forward, 5'-TCCAGCGACCTTCCTCATCC-3' and p21-reverse, 5'-CATAGCCTCTACTGCCACCATC-3'; CDK6-forward, 5'-TACCCTCTCTGCTGCTTTCAA-3' and CDK6-reverse, 5'-TGTGCTACTCATTTTGCTCACC-3'; KRAS-forward, 5'-TGTCATCTTGCCTCCCTACC-3' and KRAS-reverse, 5'-TTCTCTTGAGCCCTGAGGAA-3'; $\beta$-actin-forward, 5'-GTGGACATCCGCAAAGAC-3' and $\beta$-actin-reverse, 5'-AAAGGGTGTAACGCAACTA-3'.

The relative mRNA levels of these genes were calculated using the $2^{-\Delta \Delta C t}$ method.

Western blot analysis. Lentivirus-transduced cells were washed twice with ice-cold PBS and lysed in 2X SDS sample buffer (10 mM EDTA, 4\% SDS, 10\% Glycine in $100 \mathrm{mM}$ Tris- $\mathrm{HCl}$ buffer, $\mathrm{pH} 6.8$ ) at $4^{\circ} \mathrm{C}$ and boiled for $5 \mathrm{~min}$. The cell lysate was then centrifuged at $12,000 \mathrm{rpm}$ for $15 \mathrm{~min}$ at $4^{\circ} \mathrm{C}$ and the supernatant was collected and preserved at $-80^{\circ} \mathrm{C}$ prior to use. The protein concentration was determined using the BCA protein assay kit. Equal amount of proteins $(30 \mu \mathrm{g})$ were loaded and separated on $10 \%$ SDS-PAGE gels and transferred onto PVDF membranes (Millipore). Proteins were probed overnight at $4^{\circ} \mathrm{C}$ with the indicated primary antibodies, followed by incubation with horseradish peroxidase-conjugated second antibodies (Santa Cruz Biotechnology) at room temperature for $1 \mathrm{~h}$. Immunoreactivity was developed with enhanced chemiluminescent autoradiography (ECL kit; Amersham Biosciences).

Cell viability assay. Cell proliferation was assessed using a colorimetric assay with 3-(4,5-dimethylthiazol-2-yl)-2, 5-diphenyltetrazolium bromide (MTT). After infection for 4 days, HCT116 cells $\left(2.5 \times 10^{3}\right.$ cells/well $)$ and DLD-1 

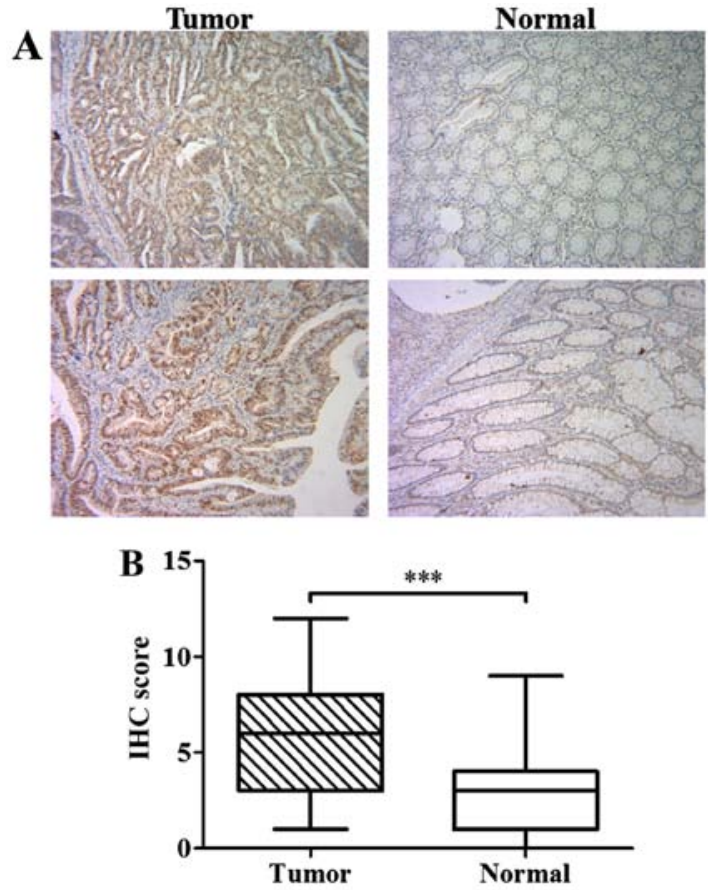

Figure 1. Immunohistochemical staining of RPS15A in normal and tumor tissues. (A) Representative immunohistochemical staining for RPS15A in normal and tumor tissues. (B) IHC score analysis revealed that the expression of RPS15A is significantly higher in tumor tissues than in normal tissues ( $\left.{ }^{* * *} \mathrm{P}<0.001\right)$. $\mathrm{P}$-value, Wilcoxon signed rank-test (matched pairs).

cells $\left(3 \times 10^{3}\right.$ cells/well) were seeded onto 96 -well plates and incubated at $37^{\circ} \mathrm{C}$ in 5 consecutive days. At each time-point, $20 \mu \mathrm{l}$ of MTT solution $(5 \mathrm{mg} / \mathrm{ml})$ was added to each well and incubated at $37^{\circ} \mathrm{C}$ for $4 \mathrm{~h}$. Then, $100 \mu \mathrm{l}$ of acidified isopropanol (10\% SDS, $5 \%$ isopropanol and $0.01 \mathrm{~mol} / 1 \mathrm{HCl}$ ) was added. The absorbance of each plate was measured at $595 \mathrm{~nm}$ using a spectrophotometer (Biotek Epoch; BioTek Instruments, Inc., Winooski, VT, USA).

Colony formation assay. In vitro tumorigenicity was determined on the basis of cell growth in a plate colony formation assay. After infection for 5 days, HCT116 and DLD-1 cells (400 cells/well) were seeded onto 6-well plates and incubated at $37^{\circ} \mathrm{C}$ for 7 days, respectively. The culture medium was changed every 2-3 days. When the colonies were formed, cells were washed and fixed, stained with crystal purple for $20 \mathrm{~min}$, and washed 3 times with $\mathrm{ddH}_{2} \mathrm{O}$, sequentially. The number of colonies ( $>50$ cells/colony) was counted using Colony Counter software. The morphology and size of the colonies was examined under a light microscope.

Cell cycle analysis. The cell cycle distribution (sub-G1, G0/G1, $\mathrm{S}$ or G2/M phase) was characterized by different DNA contents via flow cytometry. Lentivirus-transduced cells were collected by centrifugation at $1,500 \mathrm{rpm}$ for $5 \mathrm{~min}$, washed with PBS, and were fixed in $70 \%$ ice-cold ethanol solution. Then, the fixed cells were resuspended in a propidium iodide (PI)/RNase/PBS buffer for incubation in dark at $37^{\circ} \mathrm{C}$ for $30 \mathrm{~min}$. The stained cells were analyzed by a FACSCalibur II sorter and CellQuest FACS system (BD Biosciences, San Diego, CA, USA). The percentage of cells in each stage was analyzed.

Statistical analysis. In cytological experiments, all data were expressed as mean \pm SD of three independent experiments performed in triplicate. Statistical significance was conducted with the Student's t-test using SPSS 16.0 software. The Cramer's V in Pearson's chi-squared test was used to analyze the correlations between the RPS15A expression and the clinicopathological parameters. Kaplan-Meier model was utilized to perform survival curves and log-rank test was used to evaluate the intergroup differences. The Cox hazard model was performed to identify the independent factors for poor prognosis of $\mathrm{CRC}$ patients. $\mathrm{P}<0.05$ was considered to indicate a statistically significant result.

\section{Results}

RPS15A expression in CRC and corresponding normal tissues. To investigate the expression of RPS15A in CRC, IHC was performed in a total of 200 consecutive cases of tumor tissues and their corresponding normal tissues. Representative images
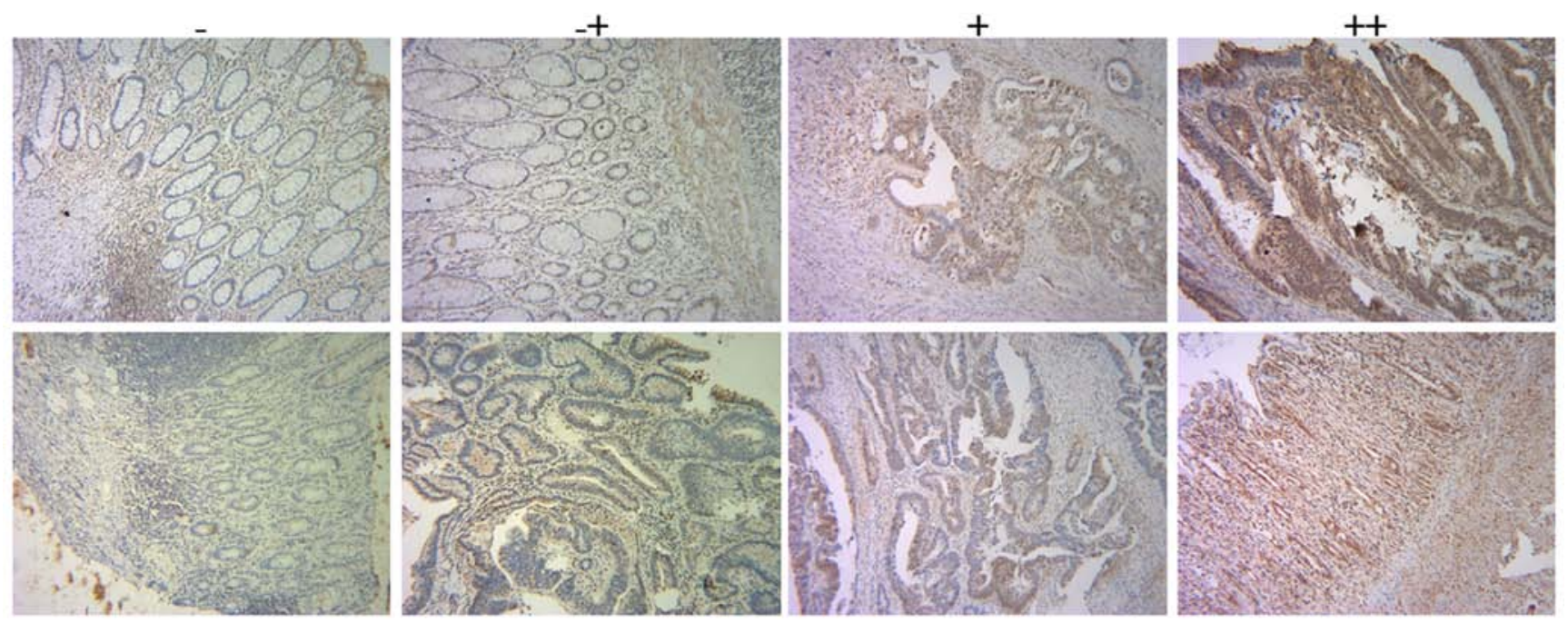

Figure 2. Representative intensity of immunohistochemical staining of RPS15A in normal and tumor tissues. (-) negative, scored 0; (-+) yellow, scored 1; (+) buffy color, scored $2 ;(++)$ reddish brown, scored 3. 
Table I. Relations between the expression of RPS15A in tumor tissues and the clinicopathological characteristics.

\begin{tabular}{|c|c|c|c|c|}
\hline & Low $(\%)(\mathrm{n}=98)$ & $\operatorname{High}(\%)(\mathrm{n}=102)$ & Correlation coefficient & P-value \\
\hline Gender & & & 0.047 & 0.509 \\
\hline Male & $58(59.2)$ & $65(63.7)$ & & \\
\hline Female & $40(40.8)$ & $37(36.3)$ & & \\
\hline Age (years) & & & 0.149 & $0.035^{\mathrm{a}}$ \\
\hline$\leq 60$ & $52(53.1)$ & $39(38.2)$ & & \\
\hline$>60$ & $46(46.9)$ & $63(61.8)$ & & \\
\hline Preoperative CEA (ng/ml) & & & 0.057 & 0.424 \\
\hline$<5$ & $62(63.3)$ & $70(68.6)$ & & \\
\hline$\geq 5$ & $36(36.7)$ & $32(31.4)$ & & \\
\hline Preoperative neoadjuvant treatment & & & 0.140 & $0.048^{\mathrm{a}}$ \\
\hline No & $80(81.6)$ & $93(91.2)$ & & \\
\hline Yes & $18(18.4)$ & $9(8.8)$ & & \\
\hline Primary tumor site & & & 0.115 & 0.269 \\
\hline Right-sided & $38(38.8)$ & $50(49.0)$ & & \\
\hline Left-sided & $26(26.5)$ & $26(25.5)$ & & \\
\hline Rectum & $34(34.7)$ & $26(25.5)$ & & \\
\hline Primary tumor size $(\mathrm{cm})$ & & & 0.040 & 0.575 \\
\hline$<4$ & $48(49.0)$ & $54(52.9)$ & & \\
\hline$\geq 4$ & $50(51.0)$ & $48(47.1)$ & & \\
\hline Primary histological type & & & 0.037 & 0.596 \\
\hline Non-mucinous & $84(85.7)$ & $90(88.2)$ & & \\
\hline Mucinous & $14(14.3)$ & $12(11.8)$ & & \\
\hline Primary differentiation & & & 0.057 & 0.422 \\
\hline Well to moderate & $59(60.2)$ & $67(65.7)$ & & \\
\hline Poor & $39(39.8)$ & $35(34.3)$ & & \\
\hline Primary pT stage & & & 0.110 & 0.121 \\
\hline $1 / 2$ & $38(38.8)$ & $29(28.4)$ & & \\
\hline $3 / 4$ & $60(61.2)$ & 73 (71.6) & & \\
\hline Primary pN stage & & & 0.189 & $0.007^{\mathrm{a}}$ \\
\hline 0 & $69(70.4)$ & $53(52.0)$ & & \\
\hline $1 / 2$ & $29(29.6)$ & $49(48.0)$ & & \\
\hline Vascular invasion & & & 0.084 & 0.237 \\
\hline No & $92(93.9)$ & $91(89.2)$ & & \\
\hline Yes & $6(6.1)$ & $11(10.8)$ & & \\
\hline Nerve invasion & & & 0.079 & 0.265 \\
\hline No & $91(92.9)$ & $90(88.2)$ & & \\
\hline Yes & $7(7.1)$ & $12(11.8)$ & & \\
\hline Synchronous distant metastases & & & 0.134 & 0.058 \\
\hline No & $82(83.7)$ & $74(72.5)$ & & \\
\hline Yes & $16(16.3)$ & $28(27.5)$ & & \\
\hline
\end{tabular}

CEA, carcino-embryonic antigen. Correlation coefficient and P-value, Cramer's V in Pearson's chi-squared test. Bold font for P<0.10; ${ }^{\mathrm{a}} \mathrm{P}<0.05$. Synchronous distant metastases were defined as a diagnosis of distant metastases together with, or within a 6-month interval, of the diagnosis of the primary colorectal cancer. The pathological tumor stage was documented according to the AJCC TNM classification (version 7, 2010).

of IHC staining of tumor and corresponding normal tissues are shown in Figs. 1A and 2. IHC score of RPS15A in CRC tissues was significantly higher than that in normal tissues $(\mathrm{P}<0.001$; Fig. 1B), suggesting that RPS15A was accumulated in CRC. 
Table II. Univariate and multivariate analysis for overall survival.

\begin{tabular}{ccccccc}
\multicolumn{2}{c}{ Univariate analysis } & & \multicolumn{3}{c}{ Multivariate analysis } \\
\cline { 6 - 7 } & $95 \% \mathrm{CI}$ & P-value & & HR & $95 \%$ CI & P-value
\end{tabular}

Gender

Male

Female

Age (years)

$\leq 60$

$>60$

Preoperative CEA (ng/ml)

$<5$

$\geq 5$

Preoperative neoadjuvant treatment

No

Yes

Primary tumor site

Right-sided

Left-sided

Rectum

0.559

$0.308-1.013$

0.326

$0.171-0.623$

0.055

0.001

0.738

0.739

0.389-1.401

0.325-1.680

0.353

Primary tumor size $(\mathrm{cm})$

$<4$

$\geq 4$

Primary histological type

1.619

$0.995-2.634$

0.052

1

1.034

0.581-1.841

0.910

Non-mucinous

Mucinous

Primary differentiation

Well to moderate

Poor

1

0.988

0.490-1.996

0.974

1
0.813

0.349-1.893

0.632

1

1.558

$0.962-2.523$

0.071

1

1.235

$0.680-2.241$

0.488

Primary pT stage

$1 / 2$

$3 / 4$

Primary pN stage

0

$1 / 2$$$
4.549
$$

2.692-7.686

$<0.001^{\mathrm{a}}$

2.009

1.078-3.744

$0.028^{\mathrm{a}}$

Vascular invasion

No

Yes

0.993

0.429-2.301

0.988

1

0.839

$0.342-2.058$

0.701

Nerve invasion

No

Yes

2.812

1.501-5.268

${ }^{-}{ }^{\mathrm{a}}$

1

Synchronous distant metastases

No

Yes

1

8.857

$5.391-14.552$

$<0.001^{\mathrm{a}}$

5.033

$2.478-10.220$

$<0.001^{\text {a }}$

Expression of PRS15Ain tumor tissues

Low

High 


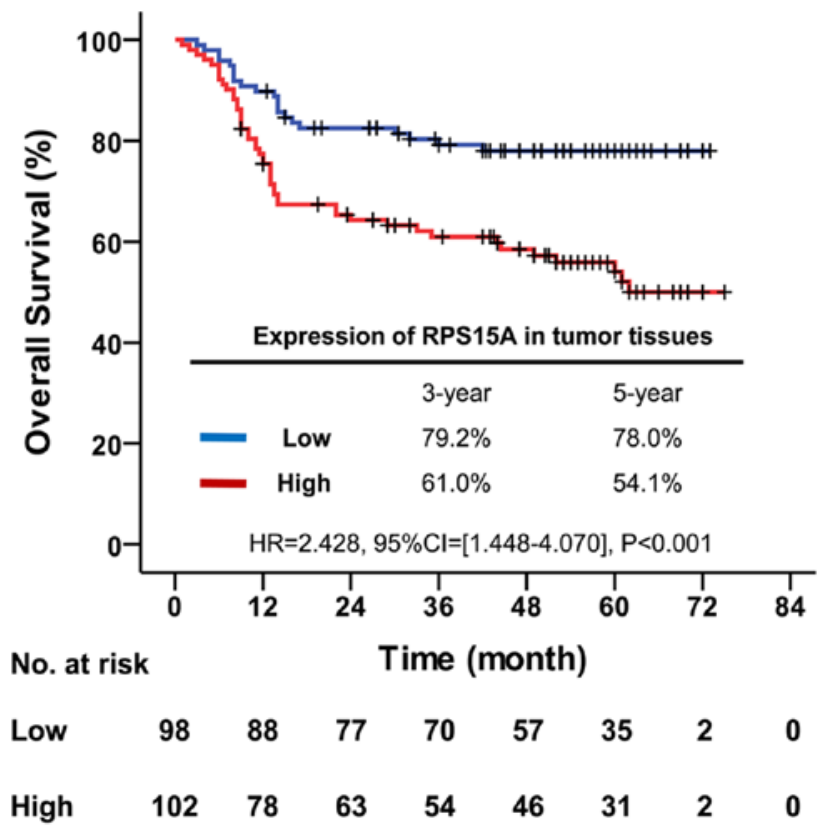

Figure 3. Kaplan-Meier survival curve analysis in colorectal cancer patients after primary tumor resection. HR, hazard ratio; CI, confidence interval; P-value, log-rank test.

Association of the RPS15A expression with clinicopathological characteristics. As shown in Table I, we summarized the correlation between RPS15A expression and clinicopathological characteristics of CRC patients. For IHC score, the median value was defined as the cut-off for the definition of high/low expression subgroups. The 200 consecutive cases of tumor tissues were analyzed and then classified into 2 groups: high expression of RPS15A ( $n=102)$ and low expression of RPS15A ( $\mathrm{n}=98$ ). High RPS15A expression was observed to be associated with older age $(\mathrm{P}=0.035)$, not receiving preoperative neoadjuvant treatment $(\mathrm{P}=0.048)$, higher primary $\mathrm{pN}$ stage $(\mathrm{P}=0.007)$, and more synchronous distant metastases $(\mathrm{P}=0.058)$. No association was observed between RPS15A expression and other clinicopathological characteristics.

Prognostic significance of RPS15A expression in CRC. To investigate the effect of RPS15A expression on the prognosis of CRC patients, the Kaplan-Meier method was used. The median follow-up time of all patients was 49.0 months (inter-quartile range, 14.0-61.0 months). The overall survival of patients with low expression of RPS15A was significantly better than patients with high expression (HR, 2.428, 95\% CI, 1.448-4.070; $\mathrm{P}<0.001)$. The overall survival rate was decreased from $79.2 \%$ in low expression to $61.0 \%$ in high expression of RPS15A at 3 years, and from 78.0 to $54.1 \%$ at 5 years, respectively (Fig. 3).

To determine whether RPS15A could be as an independent risk factor for poor prognosis of CRC patients, Cox hazard regression model was used to conduct univariate and multivariate analysis of conventional clinicopathological factors and RPS15A. According to univariate analysis results (Table II), overall survival of CRC patients was significantly correlated with RPS15A expression $(\mathrm{P}=0.001)$, together with preoperative CEA $(\mathrm{P}=0.006)$, primary tumor site $(\mathrm{P}=0.001)$,
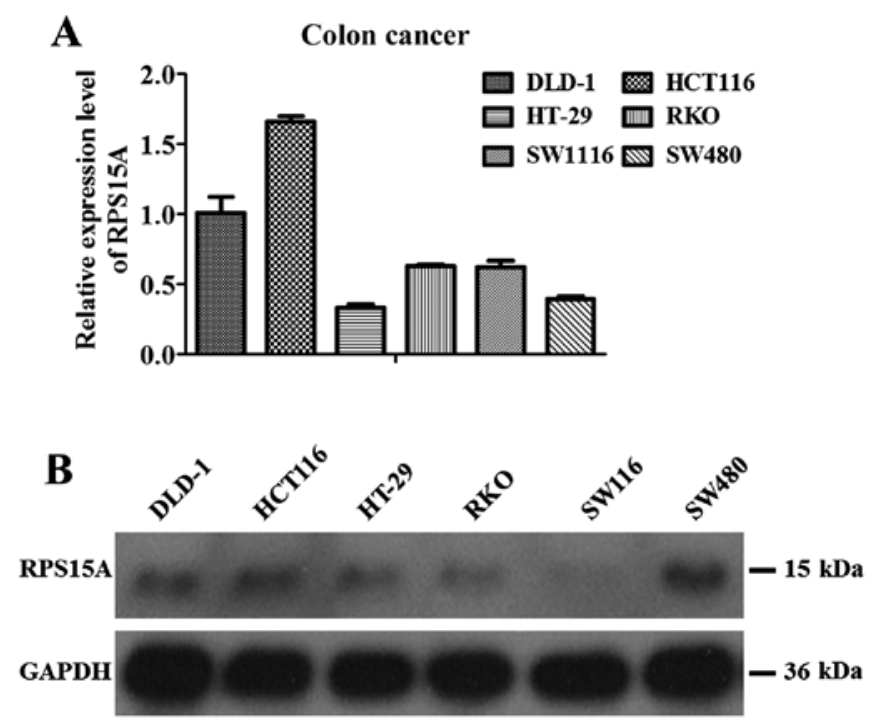

Figure 4. Expression characteristics of RPS15A in colorectal cancer cell lines. (A) Real-time PCR analysis of RPS15A mRNA in six colorectal cancer cell lines. $\beta$-actin was used as an internal gene. (B) Western blot analysis of RPS15A protein in six colorectal cancer cell lines. GAPDH was used as an internal control.

primary pT stage $(\mathrm{P}=0.001)$, primary pN stage $(\mathrm{P}<0.001)$, nerve invasion $(\mathrm{P}=0.001)$ and synchronous distant metastases $(\mathrm{P}<0.001)$. While the multivariate analysis (Table II) indicated that only RPS15A expression $(\mathrm{P}=0.022)$, primary pN stage $(\mathrm{P}=0.028)$ and synchronous distant metastases $(\mathrm{P}<0.001)$ were deemed as independent prognostic factors for overall survival of CRC patients. Therefore, high expression of RPS15A could be an independent risk factor for poor prognosis of CRC patients.

Expression levels of RPS15A in CRC cells. Real-time PCR and western blot analyses revealed that 6 human colorectal cell lines, including DLD-1, HCT116, HT-29, RKO, SW1116 and SW480, expressed RPS15A, of which highest expression was found in HCT116 cells followed by DLD-1 cells (Fig. 4A). Western blot analysis showed similar results except for the relatively higher protein level in SW480 cells (Fig. 4B). Therefore, HCT116 and DLD-1 cells were employed for further functional analysis.

To examine the role of RPS15A in CRC, infection efficiency was showed in Fig. 5A and RPS15A knockdown efficiency was confirmed by real-time qPCR and western blot analysis. Compared with NC group, the mRNA levels of RPS15A in KD-S1 groups were significantly downregulated, by 74.5 and $84.3 \%$ in HCT116 and DLD-1 cells (Fig. 5B), similar to KD-S2 (Fig. 5C). Furthermore, the RPS15A protein levels in DLD-1 cells were significantly attenuated by KD-S1 (Fig. 5D) and KD-S2 (Fig. 5E).

Functional analysis of RPS15A in CRC cells. Effect of the shRNA mediated RPS15A silencing on CRC cell viability was assessed by MTT assay. As shown in Fig. 6A and B, KD-S1 and KD-S2 inhibited HCT116 and DLD1 proliferation. Furthermore, both the number and size of colonies were observed. As shown in Fig. 6C and D, KD-S1 and KD-S2 


\section{A}
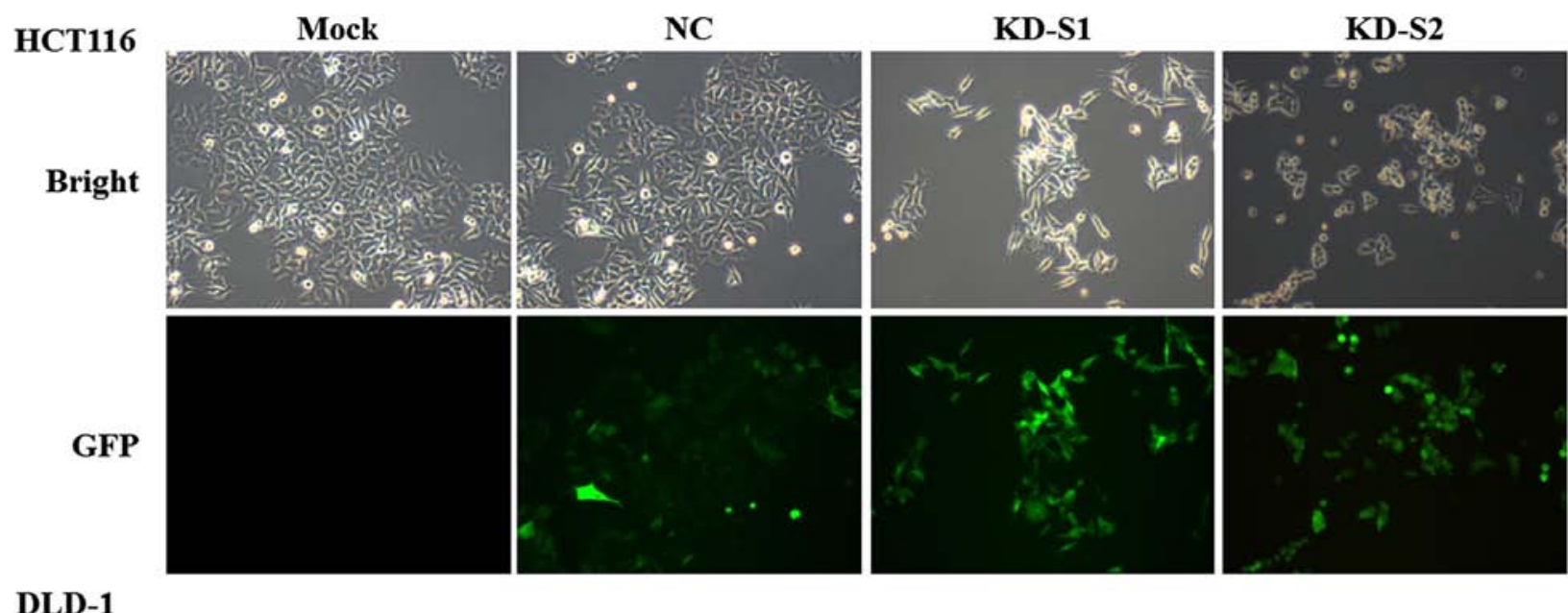

DLD-1
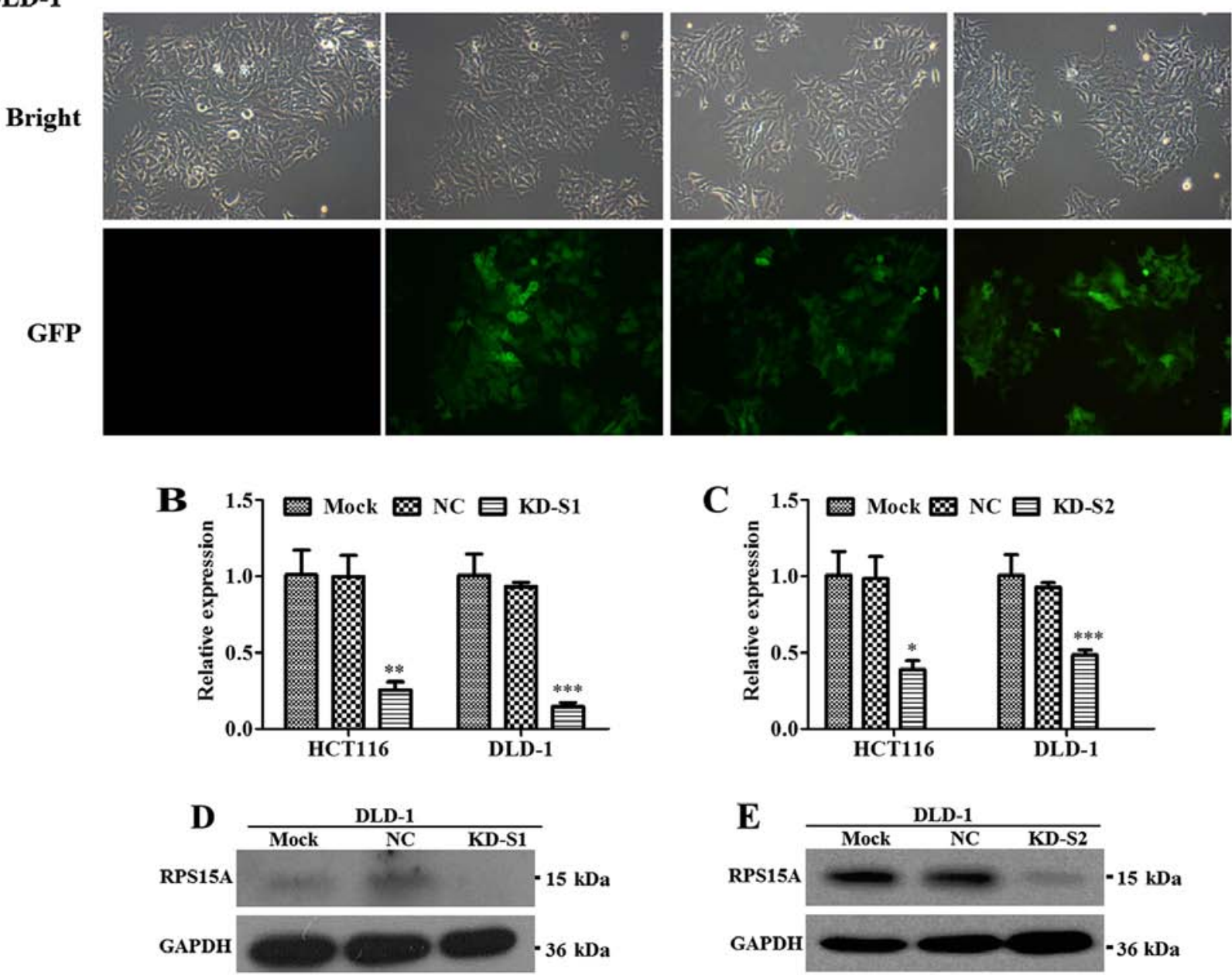

Figure 5. Knockdown efficiency of RPS15A in colorectal cancer cells. (A) Representative images of successful lentivirus infection is indicated by green fluorescence. (B) The mRNA level in HCT116 and DLD-1 after KD-S1 infection. (C) The mRNA level in HCT116 and DLD-1 after KD-S2 infection. (D) Protein level of DLD-1 after KD-S1 infection. (E) Protein level of DLD-1 after KD-S2 infection $\left({ }^{*} \mathrm{P}<0.05 ;{ }^{* * *} \mathrm{P}<0.01 ;{ }^{* * * *} \mathrm{P}<0.001\right)$

inhibited cell clone formation in HCT116 and DLD-1 cells. However, the suppression effect of KD-S2 was less compared to KD-S1 (Fig. 6E and F). Taken together, knockdown of RPS15A by shRNA was able to remarkably suppress the proliferation and colony formation.

To clarify the underlying mechanism that RPS15A silencing inhibited cell growth, flow cytometry was performed in HCT116 and DLD-1 cells (Fig. 7A and B). As shown in Fig. 7C, KD-S1 effectively blocked the cell cycle progression in HCT116. The cell population of G0/G1 phase in the KD-S1 group $(85.99 \pm 1.14 \%)$ was significantly higher than NC group $(53.10 \pm 0.56 \%$ ), whereas the cell population of $\mathrm{S}$ phase or $\mathrm{G} 2 / \mathrm{M}$ phase was much lower than NC group. Similarly, KD-S1 also blocked cell cycle progression of DLD-1 cells (Fig. 7D). The sub-G1 phase of the two CRC cell lines was not significantly changed in KD-S1 group compared with control (Fig. 7E).

In order to clarify the underlying mechanism, we conducted GeneChip analysis. According to KEGG pathway analysis of 

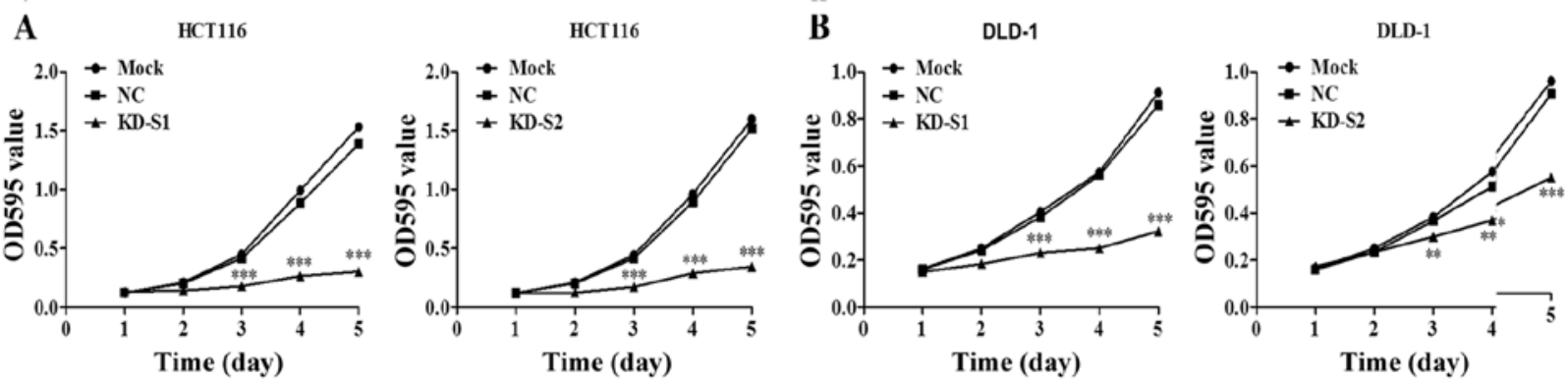

$$
\text { C }
$$

HCT116
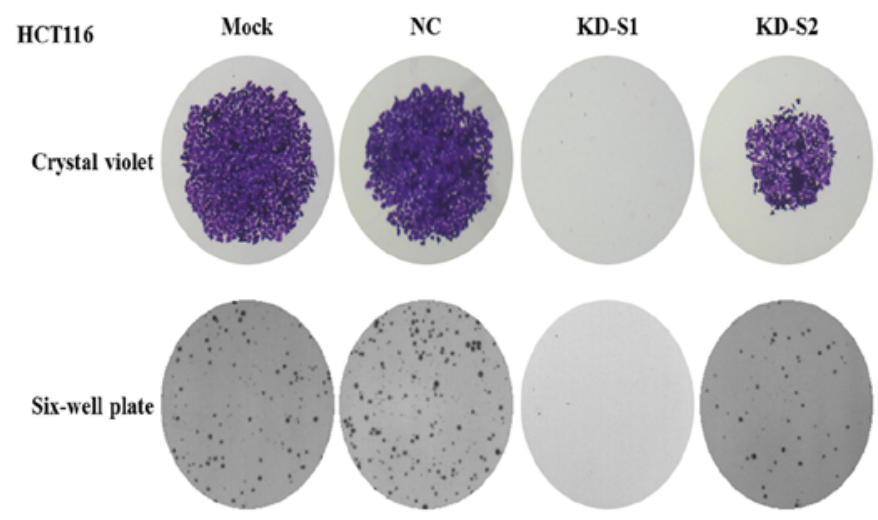

D

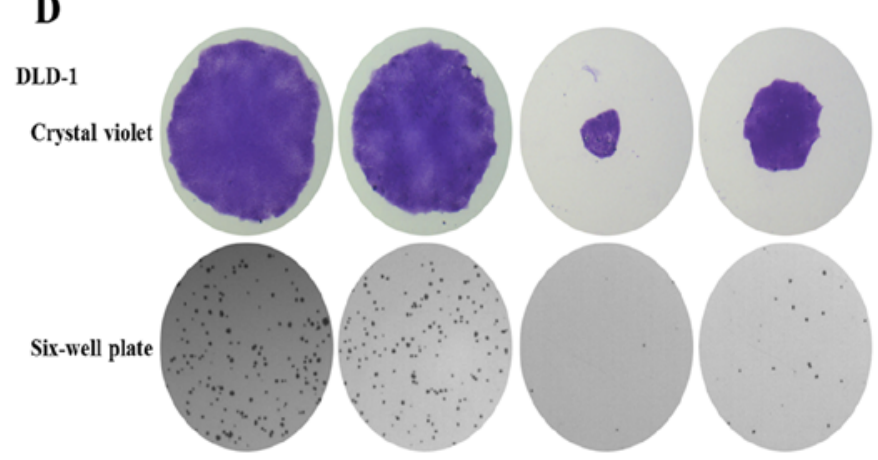

E

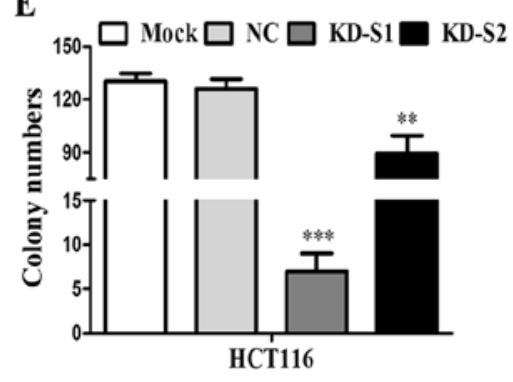

F

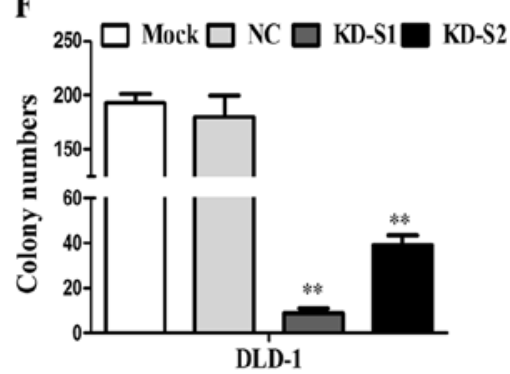

Figure 6. Effect of RPS15A knockdown on the proliferation of colorectal cancer cells. The proliferation rates of (A) HCT116 cells and (B) DLD-1 cells were significantly decreased after KD-S1 and KD-S2 infection, respectively. (C and D) Representative images of cell colony formation in HCT116 and DLD-1 cells by KD-S1 and KD-S2 infection. (E and F) Statistic results of the number of colonies in HCT116 and DLD-1 cells after KD-S1 and KD-S2 infection as shown $\left({ }^{* *} \mathrm{P}<0.01 ;{ }^{* * *} \mathrm{P}<0.001\right)$.

CRC signaling pathway, we identified that certain oncogenes and tumor suppressors were misregulated in RPS15A-KD cells (Fig. 8). We found that upregulation of p53 may correlate with an inhibited proliferation ability and cell cycle arrest in CRC cells. As validated by real-time PCR, we found that p53 was upregulated by RPS15A-KD in HCT116 cells, whereas, p21 and 14-3-3 $\alpha$ the downstream targets of p53 were strongly increased in KD-S1 group (Fig. 7F). These results indicated that knockdown of RPS15A in CRC cells led to cell cycle arrest in G0/G1 phase, which could contribute to the inhibition of CRC cell growth.

\section{Discussion}

In a retrospective study, we revealed that RPS15A was accumulated in human CRC tissues and significantly correlated with malignant clinicopathological features and a worse prognosis in CRC patients. Cell line based analysis indicated that RPS15A was widely and highly expressed in 6 human CRC cell lines, including DLD-1, HCT116, HT-29, RKO, SW1116 and SW480. Knockdown of RPS15A had a pivotal effect on CRC cell proliferation via inducing G0/G1 phase arrest rather than inducing apoptosis. GeneChip analysis suggested that RPS15A depletion might lead to misregulation of p53 signaling pathway.

Ribosome biogenesis is an essential biological process in human. To date, a number of oncogenic or tumor suppressive genes have been identified to affect the formation of mature ribosome (12-15), which suggest that protein synthesis machinery plays an important role in the regulation of malignant transformation. For instance, the oncogenic WBSCR22 protein, important for ribosome small subunit biosynthesis, is 

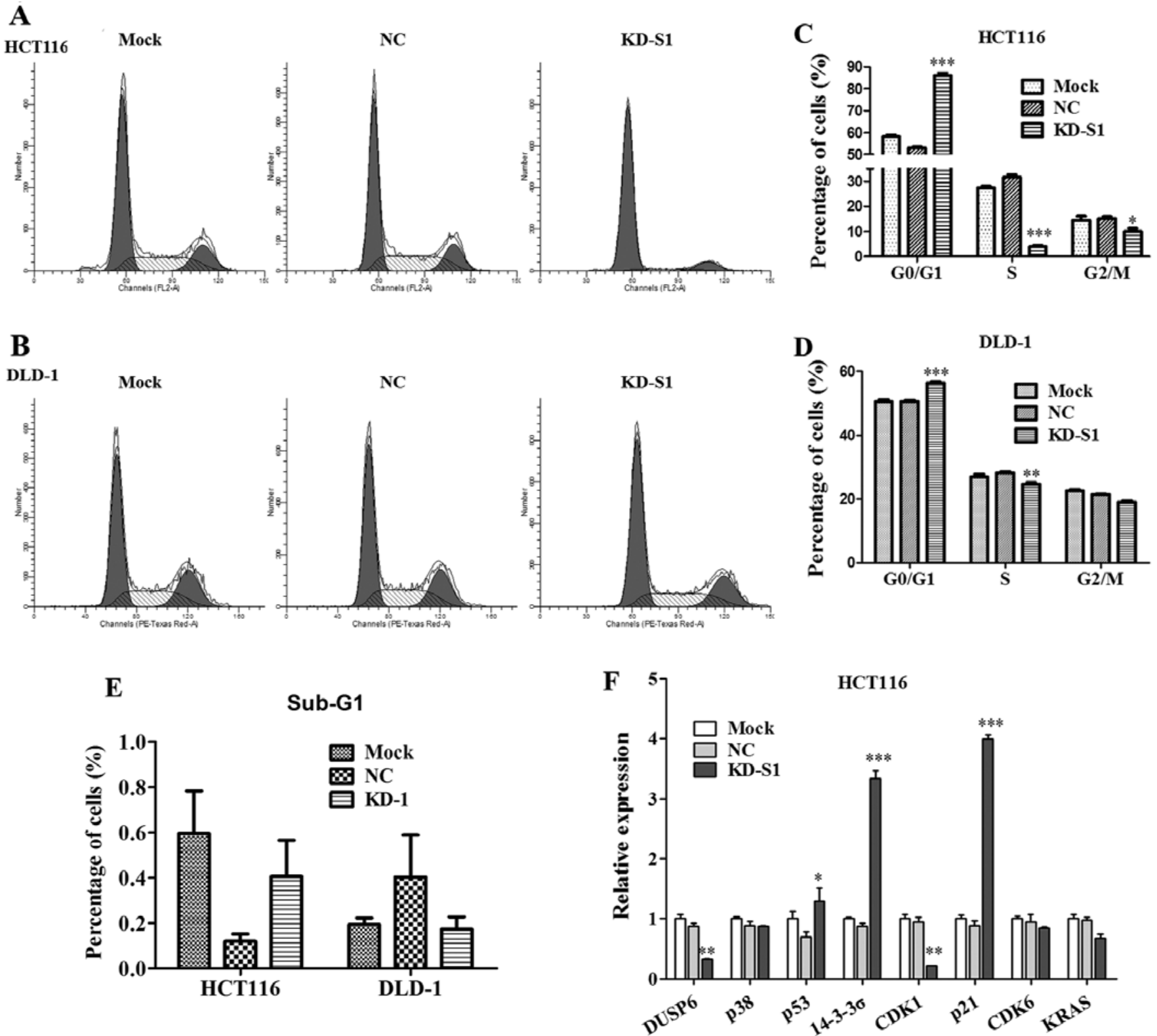

Figure 7. Effect of RPS15A knockdown on cell cycle distribution. (A and B) Flow cytometry histograms showing the cell cycle distribution patterns of HCT116 and DLD-1 cells. (C and D) Graphical representation of the percentage of cells in each phase of the cell cycle. (E) Percentage of sub-G1 phase in CRC cell lines of each group. (F) Relative quantitation at mRNA level $\left({ }^{*} \mathrm{P}<0.05 ;{ }^{* *} \mathrm{P}<0.01 ;{ }^{* * *} \mathrm{P}<0.001\right)$.

upregulated in invasive breast cancer (16). Ribosomal protein S6 (RPS6), a key regulator of 40 S ribosome biogenesis, actively participates in human esophageal cancer progression. RPS6 knockdown resulted in a reduction in esophageal cancer cell growth, migration and invasion (17).

The present study is the first reporting the potential role of RPS15A in CRC initiation and progression. In this investigation, we studied 200 paired colorectal tissue samples from 200 CRC patients, and analyzed their clinicopathological significance. As shown in Fig. 1B and the Results section, RPS15A was significantly upregulated in tumor tissues compared to normal tissues $(\mathrm{P}<0.001$, Wilcoxon signed-rank test). RPS15A was strongly stained in the enlarged nuclei in most of the tumor cells, whereas mildly stained in the cytoplasm (Fig. 1A). As a ribosomal protein, RPS15A is normally localized in the cytoplasm. However, in CRC tumor tissues, overexpression and nuclear localization of RPS15A implied an abnormal and malignant function of this protein. In addition to the abnormal expression pattern of RPS15A in tumor tissues, relatively higher expression of RPS15A predicted a lower survival rate in CRC patients (Fig. 3), strongly suggesting that RPS15A could be a potential target for early diagnosis and prevention, in addition to helping with developing novel targeted therapies for late stage disease. Consistent with our findings, RPS15A was also found to be correlated to other types of cancers, including hepatic, osteosarcoma and lung cancer $(10,11,18)$, adding RPS15A as a potential antitumor target for drug development.

According to our GeneChip analysis, the knockdown of RPS15A by small interfering RNA might lead to upregula- 


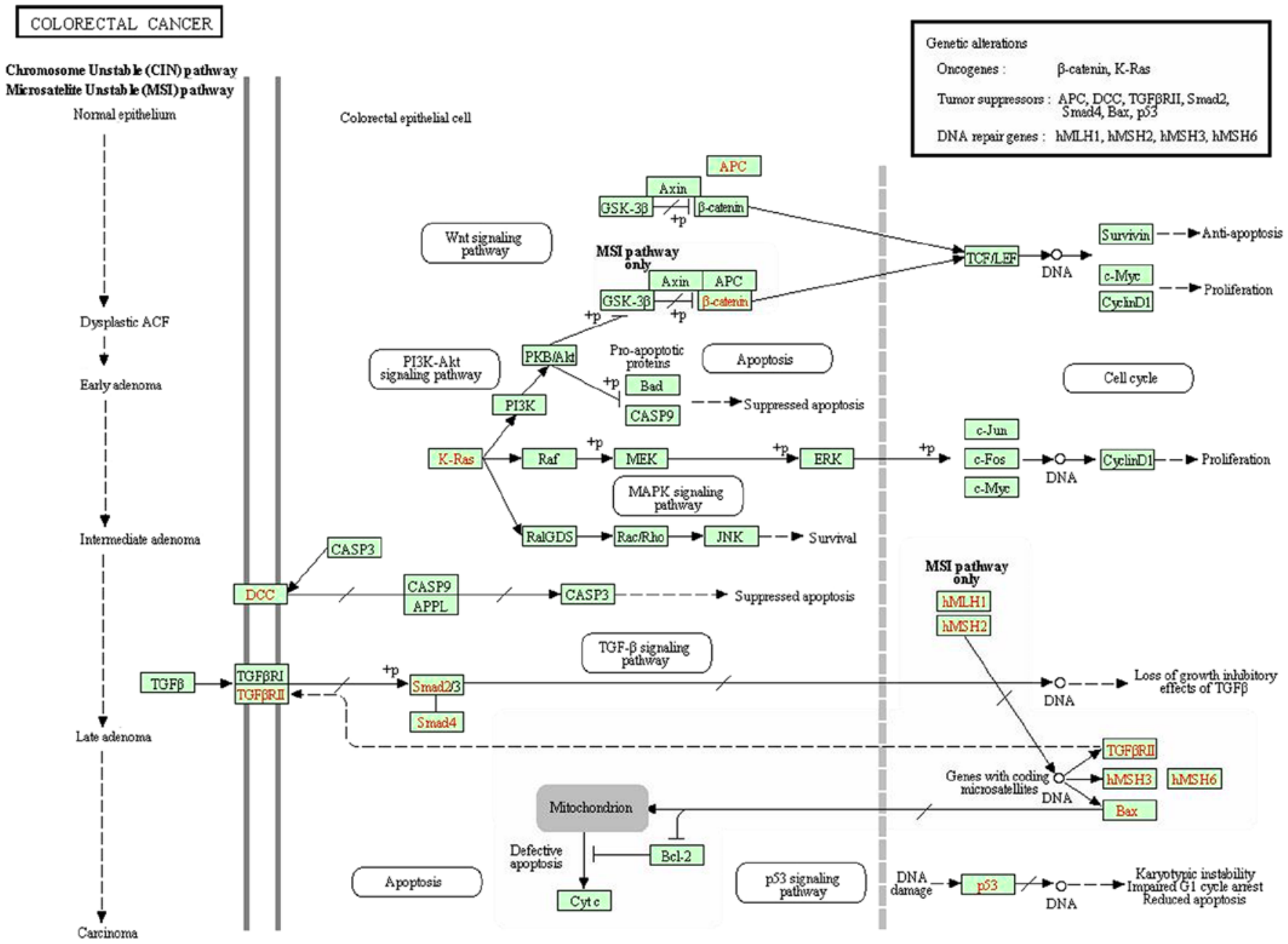

Figure 8. KEGG pathway analysis of colorectal cancer signaling pathway after RPS15A knockdown.

tion of p53. Further identification indicated that RPS15A depletion caused p21 upregulation and CDK1 downregulation, which might contribute to the cell cycle arrest at G0/G1. Upregulation of 14-3-3 $\alpha$ was also seen when RPS15A was knocked down, which might counteract the pro-apoptotic effect of the p53. This is a probable reason why knockdown of RPS15A did not induce apoptosis in the CRC cells (Fig. 7E).

Through analysis of the relation between RPS15A expression level and the clinicopathological characteristics of CRC, we found that high level of RPS15A was statistically correlated to primary $\mathrm{pN}$ stage $(\mathrm{P}=0.07)$, and showed a trend for association with synchronous distant metastases $(\mathrm{P}=0.058)$, indicating that overexpression of RPS15A might promote the mobility and invasiveness of CRC cells. However, the underlying molecular mechanism needs further investigation.

In conclusion, overexpression of RPS15A predicts a worse prognosis and outcome of CRC patients through misregulation of cell cycle progression and promotion of metastasis. Knock-down of RPS15A expression in CRC cells, remarkably induced cell growth suppression and cell cycle arrest via p21 upregulation and CDK1 downregulation. Our findings identify RPS15A as a potential therapeutic target against CRC.

\section{Acknowledgements}

The present study was supported by grants from the National Natural Science Foundation of China (no. 81272390).

\section{References}

1. Sánchez-Tena S, Vizán P, Dudeja PK, Centelles JJ and Cascante M: Green tea phenolics inhibit butyrate-induced differentiation of colon cancer cells by interacting with monocarboxylate transporter 1. Biochim Biophys Acta 1832: 2264-2270, 2013.

2. Labianca R, Nordlinger B, Beretta GD, Brouquet A and Cervantes A; ESMO Guidelines Working Group: Primary colon cancer: ESMO Clinical Practice Guidelines for diagnosis, adjuvant treatment and follow-up. Ann Oncol 21 (Suppl 5): v70-v77, 2010.

3. Wolpin BMMJ, Meyerhardt JA, Mamon HJ and Mayer RJ: Adjuvant treatment of colorectal cancer. CA Cancer J Clin 57: 168-185, 2007.

4. Uechi T, Nakajima Y, Nakao A, Torihara H, Chakraborty A, Inoue $\mathrm{K}$ and Kenmochi N: Ribosomal protein gene knockdown causes developmental defects in zebrafish. PLoS One 1: e37, 2006.

5. Nakao A, Yoshihama M and Kenmochi N: RPG: The Ribosomal Protein Gene database. Nucleic Acids Res 32: D168-D170, 2004.

6. Lambertsson A: The minute genes in Drosophila and their molecular functions. Adv Genet 38: 69-134, 1998. 
7. Oliver ERST, Saunders TL, Tarlé SA and Glaser T: Ribosomal protein L24 defect in belly spot and tail (Bst), a mouse Minute. Development 131: 3907-3920, 2004.

8. Jiménez L, Becerra A and Landa A: Cloning, expression and partial characterization of a gene encoding the S15a ribosomal protein of Taenia solium. Parasitol Res 92: 414-420, 2004.

9. Akiyama N, Matsuo Y, Sai H, Noda M and Kizaka-Kondoh S: Identification of a series of transforming growth factor betaresponsive genes by retrovirus-mediated gene trap screening. Mol Cell Biol 20: 3266-3273, 2000.

10. Xu M, Wang Y, Chen L, Pan B, Chen F, Fang Y, Yu Z and Chen G: Down-regulation of ribosomal protein S15A mRNA with a short hairpin RNA inhibits human hepatic cancer cell growth in vitro. Gene 536: 84-89, 2014

11. Zhao X, Shen L, Feng Y, Yu H, Wu X, Chang J, Shen X, Qiao J and Wang J: Decreased expression of RPS15A suppresses proliferation of lung cancer cells. Tumour Biol 36: 6733-6734, 2015.

12. Bordeleau ME, Robert F, Gerard B, Lindqvist L, Chen SM, Wendel HG, Brem B, Greger H, Lowe SW, Porco JA Jr, et al: Therapeutic suppression of translation initiation modulates chemosensitivity in a mouse lymphoma model. J Clin Invest 118: 2651-2660, 2008.
13. Hagner PR, Mazan-Mamczarz K, Dai B, Balzer EM, Corl S, Martin SS, Zhao XF and Gartenhaus RB: Ribosomal protein S6 is highly expressed in non-Hodgkin lymphoma and associates with mRNA containing a 5 ' terminal oligopyrimidine tract. Oncogene 30: 1531-1541, 2011.

14. Panwalkar A, Verstovsek S and Giles FJ: Mammalian target of rapamycin inhibition as therapy for hematologic malignancies. Cancer 100: 657-666, 2004.

15. Venticinque L, Jamieson KV and Meruelo D: Interactions between laminin receptor and the cytoskeleton during translation and cell motility. PLoS One 6: e15895, 2011.

16. Õunap K, Käsper L, Kurg A and Kurg R: The human WBSCR22 protein is involved in the biogenesis of the $40 \mathrm{~S}$ ribosomal subunits in mammalian cells. PLoS One 8: e75686, 2013.

17. Kim SH, Jang YH, Chau GC, Pyo S and Um SH: Prognostic significance and function of phosphorylated ribosomal protein S6 in esophageal squamous cell carcinoma. Mod Pathol 26: 327-335, 2013.

18. Zhang C, Zhang T, Song E, Himaya SW, Chen X and Zheng L: Ribosomal protein S15A augments human osteosarcoma cell proliferation in vitro. Cancer Biother Radiopharm 29: 451-456, 2014. 